\title{
Multi-Agent Framework Based on Semantic Approach for B2C Activity
}

\author{
K. Kannan ${ }^{1}$, K. Raja ${ }^{2}$, M. Aramudhan ${ }^{3}$ \\ ${ }^{1}$ Research Scholar, Faculty of Computer Science Engineering, \\ Sathyabama University, Chennai, India \\ ${ }^{2}$ Principal, and Professor, Department of Computer Science Engineering, \\ Dhaanish Ahmed College of Engineering, Chennai, India \\ ${ }^{3}$ Head, and Professor, Department of Information Technology, \\ PKIET, Karaikal, Pondicherry University, India \\ ${ }^{1}$ kannankphd@gmail.com \\ 2drkrajamit@gmail.com \\ 3 aranagai@yahoo.co.in
}

\begin{abstract}
Multi-Agent-Systems (MAS) appear as the most optimal solution for implementing in growing E-Commerce application for various Business-to-Customer (B2C) model activities. Multi-Agent technology along with the use of machine learning approaches increases the level of prediction accuracy. Consumer Buying Behavior (CBB) model is adopted in this proposed work, where, personal preferences (personal profiles) are rated with the statistical data at various stages, which aid the decision making process but it is rule based and produce less accuracy when the value of data cluster difference is minimal. Hence, Case Based Reasoning (CBR) model is integrated with CBB to achieve the desired level of precession and recall values, which improves the efficiency of the model. The conceptual framework with CBB and CBR is proposed, which includes multi-agent technology and machine learning process. The CBR agent based machine learning approaches increases the indexing and recall values, reduces the time complexity, and provides optimal service for the user. Through experimentation, the performance is evaluated and verified that the proposed model is better than CBB based multi-agent machine learning models.
\end{abstract}

Keyword-Multi-Agent System, Consumer Buying Behavior, Case Based Reasoning, Personal Profiles and Machine Learning

\section{INTRODUCTION}

With B2C e-commerce the desire of the customers purchasing [1] and the strategic offerings of merchants is increasing with modern bouncing trends. With the energetic growth of e-commerce application area, the Information engineering must now have to accommodate new components, concepts, techniques and meet the incoming requirements that should be accommodated very effective and smart B2C related applications, on the Internet. Because of this, MAS is gaining its advancement over traditional systems. Multi-agent technology [2], is comprised of autonomous and proactive agents that can learn, team up and participate with each other, to accomplish common objectives. The explicit growth of e-commerce services is much contributing to the increasing international trade in every product and services. Building web trust (Security and belief) along with effective performance and satisfaction is a much wanted mantra in this era.

As to make the online purchase fast, comfortable and to reduce the time the MAS technology in B2C activity in Electronic Commerce (EC) is becoming more promising now. Be that as it may, a noteworthy client shipper doubt [3] still endures in EC, for the most part in view of the nonattendance of individual contacts and to a low affirmation of the e-payment methodologies for security reasons. A multi-agent system to help B2C events of traders and clients is made out of a group of agents and a head agency. Every vendor and client is given by a software agent, dealing with a shopper individual profile [4]. The six B2C stages are well identified in the CBB model [5], where the web-based exchanging events have been built in various phases, like, Need Identification, Product Brokering, Merchant Brokering, Negotiation, Purchase and Delivery, and, Service and Evaluation. In $\mathrm{CBB}$, Customer interests with behavior are taken into account.

A normal activity during the CBB stages comprises of, a XML-based framework [4] which is used to manage agent profiles and, ACML language [6] is used for assuring portability. Ontology [7] is used for unique the portrayal of items and classifications having a place with different lists. The E-payment protocol called AIPP [8] based on existing financial Institutions is used and it is fully compliant with the standard FAST where single-use account identifiers are adopted. This approach is so significant because it rates the personal interest weights and by considering the coefficient of interests [4]. Along with CBB, CBR system is combined for making the proposed system more efficient and for better prediction. Multi-agent techniques with CBR model use the knowledge sharing process by referring the prior cases stored in the case base library. With the help of agents, the quality and retrieval values increase in the knowledge discovery process. The retriever, adapter, refiner, 
executor and evaluator agents are in place for the referring the prior cases and for reusing the best results. The best recommendations about the CBR system are predicted by the machine learning, prediction algorithm which has higher indexing and recall values. The Random Forest (RF) algorithm is adopted here for the best prediction of CBR systems.

This article is categorized as follows; Existing implementations are discussed in Section II. System Architecture is represented and discussed in Section III, and performance analyses are presented in Section IV. The conclusion is discussed in Section V.

\section{RELATED WORKS}

A number of models have been proposed for B2C activities. With due consideration, this approach is proposed by examining various papers which relate to this approach and they are presented along with this paper. Considerable literature surveys [4], [9]-[16] are made to conceptualize this framework. The advantage of the proposed MAFS approach for $\mathrm{B} 2 \mathrm{C}$ model against the other will also be presented.

As MAS and CBB are closely related [17], the CBB model remains as the backbone for various B2C activities which are supported by MAS. Only a restricted number of MAS cover a whole B2C process as codified into the CBB model [4]. Most of the systems are not interested in taking risks and so they follow the recommended financial institutions. Agents communicate through socket connections and support messagebased communication and the transactions are automated and human controlled (MAGMA and WEBS [9] [18]). The Internet-based CBB and agent-based marketplace supporting all the stages is presented in CASBA [19]. CASBA exploits Java, JavaScript, CORBA and XML technologies, whereas the publicizing is with e-mail. XML facilitates coordinating the information structures of the CASBA ontology with those of the client databases and supports the negotiation in the associated CBB stage. A 2-phase fuzzy evaluation model with a parallel dispatch model and an auction-like "one-to-multiple" negotiation model is presented in PumaMart [20]. Multi-agent systems with CBB model for promoting services in mobile networks is realized by Podobnik and Lovrek [21]. KQML is used in agent communication. An agent-based framework to facilitate a virtual shop to buyers and purchasers is presented by Al-Shrouf [11]. A distribute open multi-tiered agent systems supporting the first CBB phases of B2C processes have been presented in DAREC [10], which is characterized by its high computational efficiency and presents noteworthy points of interest as far as openness, protection, security. It allows new customized terms to be brought into the area ontology. MAST [4] [22] exploit the agent technology with CBB model, store information about a user's interests and preferences in an internal profile and weight them according to how much they actually influence the product purchase. As a result high quality, precise profiles are created when compared to the other systems which are exploited for our recommendations. Moreover MAST [23] systems are XML-based and the payment issue is taken care (CASBA and MAGMA [19] [9]) where the native commercial payment schema with the available financial institutions is used, also as presented by Podobnik [21] the communication provider effects payments as an added networking service. The MAST is fully automated and adopts a homogeneous approach to provide B2C support to the user and the selflearning CBR system [24] ease and fasten the search process and payment process. The difference with the existing system is in the context of the techniques used for rating the customer profile.

\section{MAFS APPROACH FOR B2C MODEL}

As similar to the most of the proposals, our MAFS approach for B2C Model is based on CBB, along with construction of personal profiles for the customers and merchant while performing $\mathrm{B} 2 \mathrm{C}$ activities. This approach aims for developing, designing and analyzing $\mathrm{B} 2 \mathrm{C}$ activities on the web using multi-agent for effective decision-making by adopting customized $\mathrm{CBB}$ model. In addition to that, Case-Based Reasoning (CBR) process model is integrated with $\mathrm{CBB}$ to improve the efficiency of decision-making for $\mathrm{B} 2 \mathrm{C}$ related activities on the web. With the help of an Agent Facilitator (AF), the best cases are fetched from the case base library of CBR systems. After each transaction, the Directory Facilitator (DF) is updated which is evaluated by the CBR systems for best cases, and the results are stored in the case base library. The ranking accuracy matrix of $\mathrm{CBB}$ gives the ranking list, which is evaluated in each accessing process by the evaluator agent for best prediction using random forest algorithm. As Random Forest (RF) algorithm is based on machine learning concept, it will increase the precision and recall values, hence by increasing the efficiency of the proposed model, shown in Fig. 1.

\section{A. The Random Forest Algorithm}

- Let the quantity of preparing cases is $\mathrm{N}$, and the quantity of variable in the classifier be $\mathrm{M}$.

- The variety $\mathrm{m}$ of input variables area unit accustomed confirm the choice at a hub of the tree; $\mathrm{m}$ ought to abundant but $\mathrm{M}$.

- Pick a training set for this tree by choosing $\mathrm{N}$ times with substitution from all $\mathrm{N}$ possible instructing cases. Utilize the rest of the cases to gauge the mistake of the tree, by foreseeing their classifications. 
- For every hub of the tree, every \{which way\} select $m$ variables on which to base the decision at that hub. Derive the most effective split supported these $\mathrm{m}$ factors inside the instructing set.

- Each tree is fully-developed and not trimmed, In the event that a dataset $T$ contains cases from $n$ categories, Gini Index, Gini (T) is outlined as:

$$
\operatorname{Gini}(\mathrm{T})=\sum_{j=1}^{n}\left(P_{j}\right)^{2}
$$

The proposed framework is presented in the Fig. 2. The personal preferences, personal profiles, and its various co-efficient are rated and considered in decision-making by taking into account of the previous case history. Decision-Maker's (DM's) memory is augmented by giving access to an extensive accumulation of cases called the case library [25] by quickly reviewing the most important cases which in turn support the decision process using DSS (Decision Support System). The recommendations are so prominent because of its machine learning capability. The various agents associated with the CBR used in retrieving, comparing, refining, executing and evaluating the cases of CBB stages.

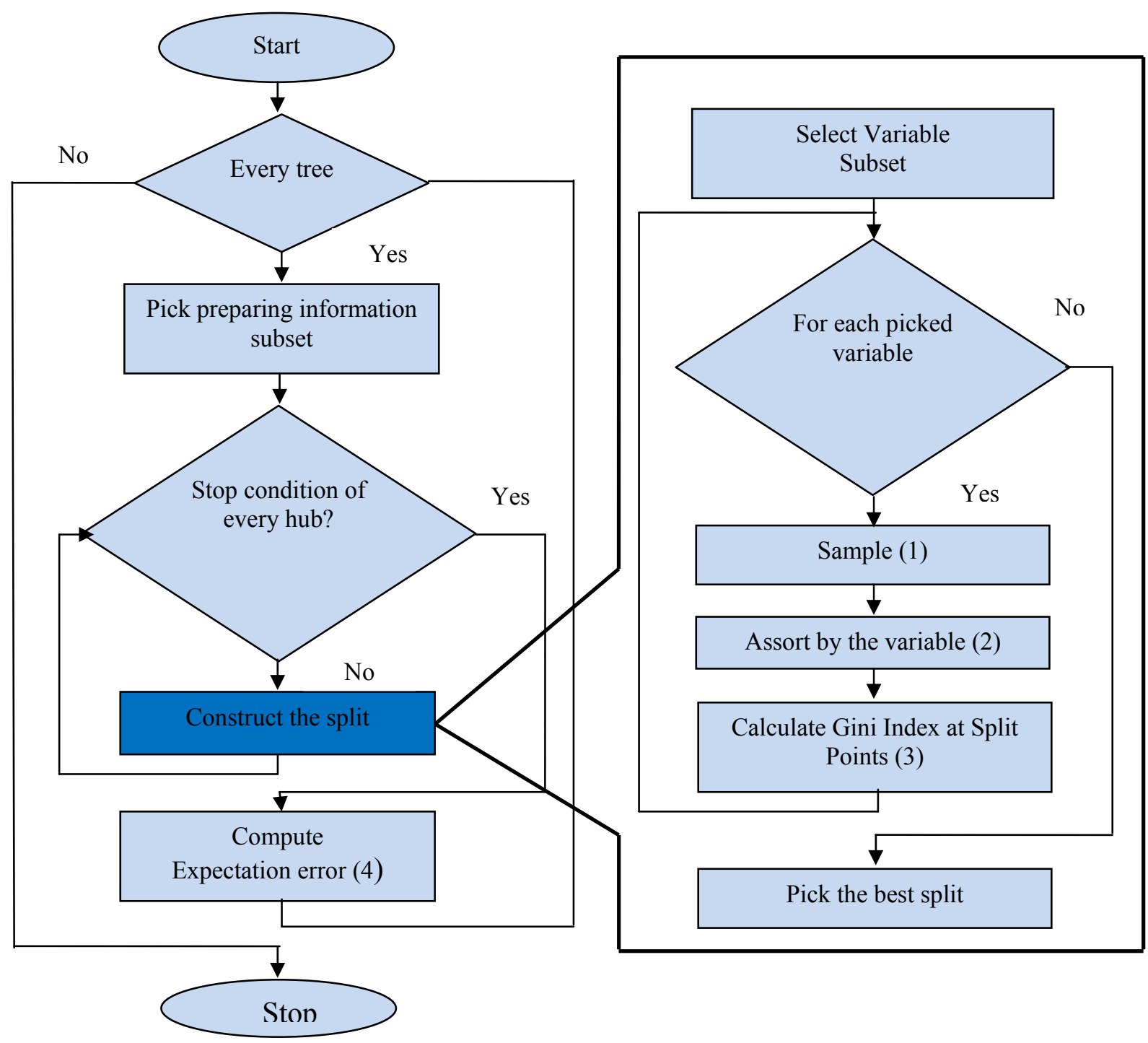

Fig. 1. Random Forest Algorithm

\section{B. The Operation and Evaluation of MAFS Model}

During the initial stage the customer comes to online [26] through the EC site initialization for a purchase and so the customer and the merchant agents are activated with due logging of the web session and in the same way, it will be deactivated when the web session ends. Generally, the agent sends its address to the agency in order to update the Yellow Page structure also, after each access to an EC site, it updates its profile, the product last access date and its weights in the directory facilitator. If the purchase is done by a new customer/merchant a 
new profile is created and the agency knowledge is updated. Every product selection, merchant selection and if the purchase is confirmed the financial institution and customer feedback is updated in the DF. An agent facilitator with the CBR system helps in every product, merchant selection by evaluating the customer profile. For this, the retriever agent check for the customer or merchant profile case history for the recommendations (if needed), if not a discovered case is recorded in the case base library. The profile consists of three coefficients, namely CI, PI and MI, where the client's worldwide enthusiasm for an item class is CI, the specific product instance on a product category is PI, and the interest about the merchant is MI. The agent also filters the merchant offers and generates one-time account number and updates the agency knowledge periodically.

The refiner agent can be utilized for refining the customer in negotiation phase which is not implemented in the proposed approach. The executor agent is invoked on purchase confirmation which in turn utilizes the web composition service for this payment operation using AIPP with the available financial institution services which are available over the web.

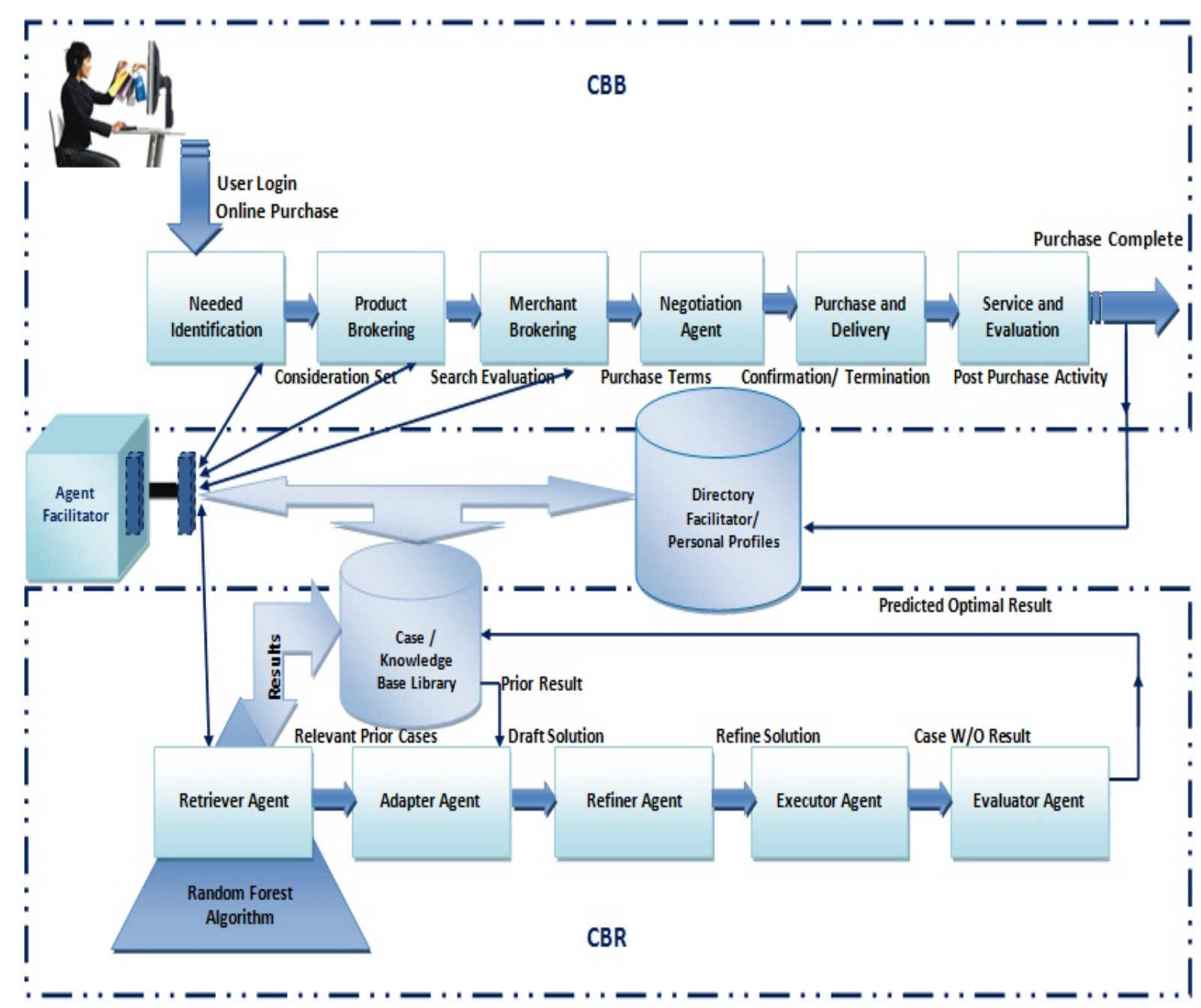

Fig. 2. The MAFS Approach for B2C Model Framework

The commonly utilized e-payment framework is the credit card [27] and card number is provided on the merchant site and so proposed known financial institutions is included in the EC specific applications for security purpose which is realized completely with secure software and hardware. In Need Identification Stage the profile is selected only for the customer product category having a CI coefficient greater than CW [4], also this is the same in the Product Brokering and Merchant Brokering stage, respectively, PI and MI are computed. $\mathrm{CI}$ is computed on the basis of the whole customer's access history in various stages s (where $\mathrm{s}=1$ to 6 ) of the specific CBB activity with the computation of the case base value (cbv) which range from 0 to 1 in which 0 is the best case and 1 is the worst case.

When a customer visits an EC site associated with the framework, as a first activity both the agents of the customer and merchant update the respective Agent Repository (AR). The AR update is retrieved by the retrieve agent, and it is updated in the case base. During each product selection and merchant selection, the case base is referred and the recommendation is provided. 
CInew $=(\mathrm{CWnew} / \mathrm{cbv})(1-\mathrm{M})$ if $\mathrm{s}=6$ where cbv is the case base value.

CInew $=($ CWnew $/ 1+\mathrm{cbv})$ where

$\mathrm{CWnew}=\{(1-\mathrm{M}) \cdot(\mathrm{CWold} / 1+\ln (1+\mathrm{LCA}-\mathrm{CD}))+\mathrm{M} \cdot \mathrm{Ks}\} \quad$ if $\mathrm{s}=1$ to 5

$\mathrm{CWnew}=\{\mathrm{CWold}+\mathrm{M} . \mathrm{Ks}\}$ if $\mathrm{s}=6$,

Where CW Category Weight is the global interest of its user product category [4] which is in the range [0;1], M Memory is a actual value extending from [0;1], LCA the Last Category Access (date). In the Service and Evaluation (i.e., $\mathrm{s}=6$ ) if the customer is unsatisfied the parameter Ks is set a value between $[-1 ; 0]$ where Ks = 1 is the minimum degree of satisfaction. The sum of the five Ks parameters has to be equal to 1 that is $\Sigma \mathrm{s}=1$ to 5 $\mathrm{Ks}=1$. The temporal distance of the last access to the product category, expressed in the day, the current $\mathrm{CW}$ value is decreased. Further, the value is reduced to the computation of CInew with the due computation of cbv value as shown below.

The context-aware, adaptable and disseminated CBR[27] approach for the service choice computes [28] the cbv value [15] using the OWL ontology and SWRL rules. The score for each retrieved experience is calculated by considering the factors like recency, similarity, and satisfaction. Recency (REi) is the new encounters favored over old encounters since they are probably going to hold again sooner rather than later thus each experience is doled out a recency value. The more up to date the experience, the bigger the recency value. Similarity (SIi) is a factor that measures the closeness of the present request with the inspected understanding and its value runs in the vicinity of 0 and 1 , where 0 signifies the aggregate contrast and 1 indicate indistinguishable requests. Fulfillment (STi) is a vital factor that measures how fulfilled the present customer agent would be, had it experienced the analyzed experience itself. The shopper assesses the provided service contingent upon its present service request and its own particular fulfillment criteria and acquires its normal level of fulfillment. These elements are computed to have the cbv (case base value) cbv = REi x SIi x STi.

After computing the scores it is used in the computation of CInew, where cbv with the highest score is the best selection case 1 and the worst selection is 0 . The results are used in our profile rating process. Experimental results are tabulated. Once the purchase is finalized the payment occurs by exploiting the AIPP protocol where payer and payee characters are verified by the particular money related establishments amid their online accounts gets to (generally with User ID and secret key over an SSL Internet session payments happen straightforwardly among the financial association) with the one use account identifiers which guarantee customers and merchants. If the payment is not happening and the purchase activity is terminated, it is updated in the case base also the agency knowledge is updated. The exactness of an expectation algorithm, to be specific precision metrics, characterization exactness metrics, and rank exactness metrics [16] are taken into account. Predictive accuracy metrics using random forest measure how to shut the prescribed frameworks anticipated the evaluations to the genuine client ratings.

\section{IV.PERFORMANCE ANALYSIS}

The processed results are tabulated in Table I, and shown in Fig. 3, CI with attributes like sample cbv, days and $\mathrm{CBB}$ [29] stages s. Considering the case $\mathrm{s}=1$ where $\mathrm{CWold}$ value $=0.9, \mathrm{ks}=1$ and the memory $\mathrm{M}=0.1$. With the decrease in the number of days and cbv value the CInew increase, this shows the high rated value.

TABLE I. Evaluation of Case Base Value with Customer Interest where $s=1$ to 5

\begin{tabular}{|c|c|c|c|}
\hline Sl. No. & No of days & Cbv Value & CInew \\
\hline 1 & 5 & 0.9 & 0.55 \\
\hline 2 & 5 & 0.8 & 0.62 \\
\hline 3 & 2 & 0.9 & 0.72 \\
\hline 4 & 2 & 0.8 & 0.81 \\
\hline
\end{tabular}

In the same way for $\mathrm{s}=6$ the value is calculated and it has shown in Table II, and Fig. 4 , with memory $=0.1$, CWold $=0.97$ and $\phi=-1$, in which, if cbv value decreases the CInew increases which are the highest rated value.

TABLE II. Evaluation of Case Base Value with Customer Interest where $s=6$

\begin{tabular}{|c|c|c|}
\hline Sl. No. & Cbv Value & CInew \\
\hline 1 & 0.9 & 0.45 \\
\hline 2 & 0.8 & 0.48 \\
\hline 3 & 0.7 & 0.51 \\
\hline
\end{tabular}




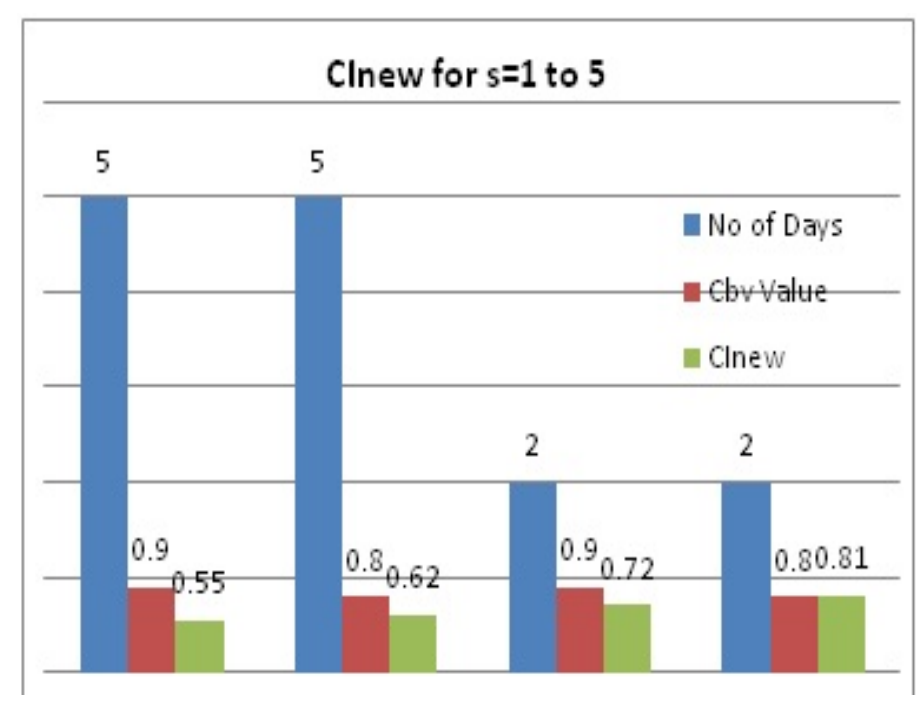

Fig. 3. Comparison of Case Base Value with Customer Interest where $s=1$ to 5

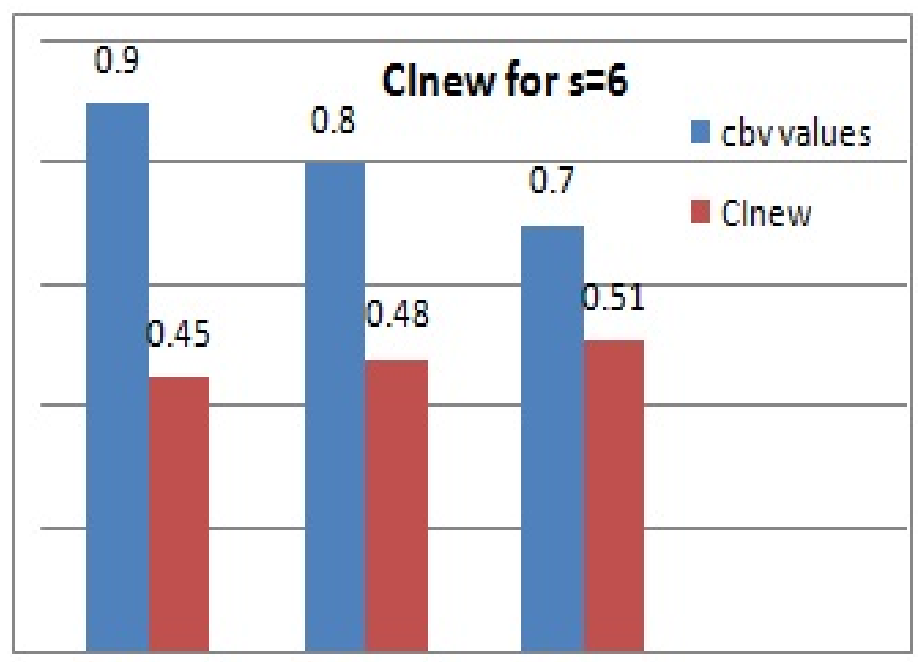

Fig. 4. Comparison of Case Base Value with Customer Interest where $s=6$

\section{CONCLUSION}

This paper presents the MAFS approach for B2C which integrates multi-agents with CBB and CBR, which is a XML-based framework to help clients and dealers in an incorporated and customized path, assessing their own advantages and personal preferences based on the behaviors appeared amid their B2C exercises, as illustrated in CBB model. This builds a high-quality user profile, adopts a safe, unified payment conspire in view of existing Financial Institutions and account numbers. The experimentation is carried out using the JADEbased system. In future, the refiner agent can be implemented for refining the customer in the negotiation phase of $\mathrm{CBB}$, which is not implemented in the proposed approach. Also, new prediction algorithm will be proposed to make the CBR system to have more prediction accuracy to make the proposed system much more optimal, secure, elegant and fast.

\section{REFERENCES}

[1] Durmaz et al., "Integrated Approach to Factors Affecting Consumer Purchase Behavior in Poland and an Empirical Study," Global Journal of Management and Business Research, Vol.12, Iss.15, Ver.1.0, pp.53-79, 2012.

[2] Costina, B., Zoranb, B., Hans-Dieterc, B., Mirjanab, I., "Software agents: languages, tools, platforms," Computer Science and Information Systems, Vol.11, Iss.8, pp.255-298, 2011.

[3] Iglesias-Pradas, S., Pascual-Miguel, F., Hernndez-Garca, Chaparro-Pelez, J., "Barriers and drivers for non-shoppers in B2C ECommerce: a latent class exploratory analysis," ACM Journal, Computers in Human Behavior, Vol.29, Iss.2, pp.314-322, 2013.

[4] Rosaci, D., Sarnè, G.M.L, "Multi-agent technology and ontologies to support personalization in B2C E-Commerce," Elsevier, Electronic Commerce Research and Applications, pp.13-23, 2013.

[5] Gordon R. Foxall, Jorge M. Oliveira-Castro, Victoria K. James, Teresa C. Schrezenmaier, "Consumer Behaviour Analysis," Durham Research Online, Durham University, UK, pp.1-9, 2011.

[6] Grosof, B.N., Labrou, Y., "An approach to using XML and a rule-based content language with an agent communication language," Issues in Agent Communication, Springer, LNCS, Vol.1916, pp.96-117, 2000. 
[7] De Meo, P., Quattrone, G., Rosaci, D., Ursino, D., "Bilateral semantic negotiation: a decentralized approach to ontology enrichment in open multi-agent systems," International Journal of Data Mining, Modelling, and Management, Vol.4, Iss.1, pp.1-38, 2012.

[8] Garruzzo, S., Sarnè, G.M.L., Palopoli, L., "AIPP: a FAST-complied e-payment protocol," inProc. of the IADIS International Conference -Applied Computing-IADIS'06, IADIS Press, pp.406-410, 2006.

[9] Shamir, A., "Secure Click: a web payment system with disposable credit card numbers," Springer, Financial Cryptography, in Proc. of the 5th International Conference, FC 2001, Grand Cayman, British West Indies, pp.232-242, 2001.

[10] Tsvetovatyy, M. B., and Gini, M, "Toward a virtual marketplace: Architectures and strategies," in Proc. of the 1st International Conference on the Practical Application of Intelligent Agents and Multi-Agent Technology, pp.597-613, 1996.

[11] Palopoli, L., Rosaci, D., Sarnè, G.M.L., "A Multi-tiered recommender system architecture for supporting e-commerce," in Proc. of the 6th International Symposium on Intelligent Distributed Computing - IDC 2012, Springer, Calabria, Italy, Vol.446, pp.71-81, 2012.

[12] Al-Shrouf, F., Turani, A., Al-Shqeerat, K., "Software agents for E-Commerce data workflow management," inProc. of the Second International Conference, ICSECS-2011, Springer, Kuantan, Pahang, Malaysia, Vol.180, pp.96-106, 2011.

[13] Maes, P., "Agents that reduce work and information overload," Communication of the ACM, Vol.37, Iss.7, pp.30-40, 1994

[14] Sierra, C., Dignum, F., "Agent-mediated electronic commerce: scientific and technological roadmap," inProc. of Agent Mediated Electronic Commerce, ACM, The European Agent Link Perspective, pp.1-18, 2001.

[15] Ye, Y., Liu, J., Moukas, A., "Agents in electronic commerce," ACM Journal, Electronic Commerce Research-Special issue on agents in electronic commerce, Vol.1, Iss.1-2, pp.9-14, 2001.

[16] Murat et al., "A Comparative Study of Reasoning Techniques for Service Selection," in Proc. of 5th International Workshop on Agents and Peer-to-Peer Computing, Springer, Hakodate, Japan, pp. 123-134, 2006.

[17] Herlocker, J.L., Konstan, J.A., Terveen, L.G., Riedl, J.T., "Evaluating collaborative filtering recommender systems," ACM Transactions on Information Systems, Vol.22, No.1, pp.5-53, 2004.

[18] Firoozeh et al., "The effect of Brand Equity on Consumer Buying Behavior in term of FMCG in Iran," Interdisciplinary Journal of Contemporary Research Business,Vol.4, No.9, pp.945-957, 2013.

[19] Lau, R., Hofstede, A., and Bruza P, "Adaptive profiling agents for electronic commerce," in Proc. of the 4th CollECTeR Conference on Electronic Commerce, Breckenridge, Colorado, 2000.

[20] Vetter, M., Pitsch, S., "Towards a flexible trading process over the Internet," Agent Mediated Electronic Commerce, The European Agent Link Perspective. LNCS, Springer, Vol. 1991, pp.148-162, 2001.

[21] Wang, Y., Tan, K.L., Ren, J., "Puma Mart: A parallel and autonomous agent-based Internet marketplace," Electronic Commerce Research and Applications, Vol.3, Iss.3, pp.294-310, 2004.

[22] Podbnik, V., Lovrek, I., "Multi-agent system for automation of B2C process in the future internet," in Proc. of the IEEE Conference on Computer Communication Workshops (INFOCOM 2008), pp.1-4, 2008.

[23] Rosaci, D., "Exploiting agent ontologies in B2C virtual marketplaces," Journal of Universal Computer Science, Vol.11, Iss.6, pp.1011-1039, 2005.

[24] Rosaci, D., Sarnè, G.M.L., "A multi-agent recommender system for supporting device adaptivity in e-commerce," ACM, Journal of Intelligent Information System, Vol.38, Iss.2, pp.393-418, 2012.

[25] Mobyen et al., "Physical Activity Identification using Supervised Machine Learning and based on Pulse Rate," International Journal of Advanced Computer Science and Applications, Vol.4, No.7, pp.209-217, 2013.

[26] Doulaverakis et al., "Galen OWL: Ontology-based drug recommendations discovery," Journal of Biomedical Semantics, Vol.3, Iss.14, 2012.

[27] Ankolekar, et al., "DAML-S: Web Service Description for the Semantic Web," in Proc. of the First International Semantic Web Conference-ISWC02, 2002.

[28] Phylis et al., "Consumers and credit card : A review of the empirical literature," Journal of Management and Marketing Research, pp.1-26, 2012.

[29] Philipp et al., "Exploiting ontology lexica for generating natural language texts from RDF data," in Proc. of the 14th European Workshop on Natural Language Generation, Association for Computational Linguistics, pp.10-19, 2013.

\section{AUTHOR PROFILE}

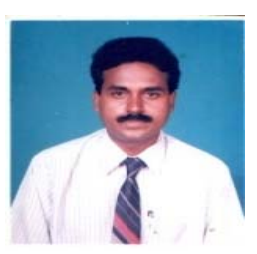

K. Kannan is a Research Scholar, Faculty of CSE, Sathyabama University, Chennai. He is presently working as an Associate Professor in Department of IT, Adhiparasakthi College of Engineering, Kalavai, Tamilnadu, India, since July 2008. He has completed his B. Sc., (CS) from Bharathiar University, 1991, M. Sc., (CS) from Bharathidasan University, 1994, M. E., (CSE) from Anna University, Chennai, India, 2008. He is a Life Member in professional bodies like ISTE - Indian Society for Technical Education, and Professional Member of ACM - Association for Computing Machinery. He has earned 19 Years of Academic Experiences from various reputed educational Institutions. He has published research papers in peer-reviewed, reputed7 International Journals, 6 National Journal, and presented the papers in 25 International (Singapore, Doha Qatar, and India), and National Conferences. His areas of interest are Knowledge and Data Engineering, Decision Support Systems, Service Oriented Architecture, Web Technology Data Structures, and Data Warehousing.

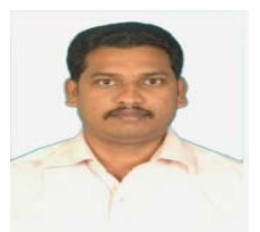

Dr. K. Raja is working as Principal at Dhaansih Ahmed College of Engineering, Chennai, India, since Sep 2016. He completed his B. Sc (Mathematics) from Madras University,1989, B.E (Computer Science and Engineering) and P.G. Diploma in Personnel Management from Annamalai University, 1993, M.B.A from Madurai Kamarajar University, 1997, M. E (Computer Science and Engineering) from Madras University, 2001, Ph. D degree in Knowledge-based systems from Sathyabama University, India, 2006. M. Phil (Human Resource Management) from Annamalai University, 2007. MLIS (Master of Library Information Science) in the year 2011 from Annamalai University. He is a life member of various professional bodies like Institution of Engineers India, Computer Society of India, and Indian Society for Technical Education, International 
Association of Computer Science and Information Technology etc. He has more than two decades of teaching experience. He has published research papers in peer-reviewed, reputed 45 International Journals, 8 National Journals, 54 International (Singapore, Malaysia, Sri Lanka, Doha Qatar, and India), and National conferences. He has written 5 books in various fields. He is a reviewer in various International, and National Journals. 2 Ph.D. Candidates are awarded their degree under his Supervision. He is supervising more than 7 Ph.D. and 2 M. Phil candidates. His areas of interest are Knowledge-Based Systems, Knowledge Management, Technology Management, Computer Networks, System Software, Software Engineering, Network Security and Data Structures and Algorithms, HR and Quality Systems. Dr. K. Raja has over 23 years of teaching experience having served in academic as well as administrative and management roles with several leading Engineering Colleges in Tamil Nadu including a term at Madras Institute of Technology, Anna University.

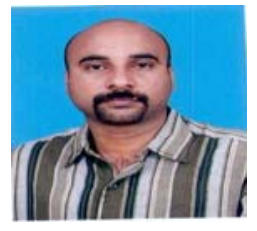

Dr. M. Aramudhan working as Head, and Professor, Department of Information Technology, Perunthalaivar Kamarajar Institute of Engineering and Technology, Karaikal, Puducherry, India. He has obtained his UG and PG in Computer Science and Engineering degree from Regional Engineering College, Tiruchirappalli, and Ph.D. from Anna University, Chennai. He has published more than 140 research articles in peer-reviewed, reputed International and National Journals and received four times best paper awards. $\mathrm{He}$ has authored a textbook. 15Ph.D. Candidates are awarded their degree under his Supervision. He has over 20 years of Academic experience. His areas of interest are Knowledge-Based Systems, Computer Networks, System Software, Software Engineering, Network Security and Data Structures and Algorithms, Service Oriented Architecture. 